Apidologie, 1978, $9(3), 195-202$.

\title{
CONTROLE GÉNÉTIQUE DES HYBRIDES INTERRACIAUX D'APIS MELLIFICA L.
}

\author{
Genetische Kontrolle der Hybriden zwischen \\ verschiedenen Rassen von Apis mellifica $L$.
}

\author{
J. M. CORNUET \\ I. N.R. A. Station expérimentale d'apiculture
}

84140 Montfavet

\begin{abstract}
SUMMARY
GENETICAL CONTROL OF INTERRACIAL HYBRIDS OF APIS MELLIFICA L.
\end{abstract}

Several authors have pointed out the advantage of creating hybrids between some races in order to increase the honey production. The best results are usually obtained with three-ways hybrids whose queen is an hybrid of two races and is inseminated by drones of a third race. But the use of such hybrids may lead to a genetical pollution in the local population of honeybees. In order to refrain this pollution as much as possible, a solution is examinated which involves two mutations making it impossible for the drones to inseminate queens naturally. These two mutations should be strongly linked, recessive and introduced in the hybrid queen in a repulsive heterozygote state. So the mother of this queen must be homozygote for one mutation and inseminated by drones sharing all the other mutation.

The pearl and cream mutations appear to fulfil these conditions. Their use should divide the number of undesirable drones by 600 . There is still to verify that when honeybees are heterozygote at these loci, their physiology is not disturbed as it has been found for two other mutations (snow and charlreuse) acting on the same ommochrome pathway.

\section{RÉSUMÉ}

Pour lutter contre la pollution génétique que peut entrainer l'utilisation de reines hybrides de races etrangères, l'auteur propose l'usage des deux mutations pearl et cream. Si une reine hybride est hétérozygote pour ces deux mutations et si celles-ci sont en opposition, les màles de sa descendance ayant une vi- 
sion normale et donc capables de féconder naturellement d'autres reines seront dans la proportion approximative de 1 pour 600 (les autres portant au moins l'une des mutations et donc aveugles). Toutefois, il reste à s'assurer qu'à l'état hétérozygote ces mutations n'ont pas d'action néfaste sur le reste du métabolisme.

\section{INTRODUCTION}

Parmi les méthodes d'amélioration génétique de la production de miel, la création d'hybrides entre races géographiques d'Apis mellifica $L$. présente un intérêt incontestable. RutTNER (1968) a dressé une liste des essais de croisements interraciaux effectués à cette date. Les résultats montrent que dans les meilleurs cas, les hybrides produisent deux fois plus de miel que les colonies locales. Depuis les expériences sur les hybrides se sont accumulées (Bornus 1972, Melnitchenko et TRICHINA 1976, Frestaye, Eavie -et Boesiger 1974, VelitchKov 1976) et le plus souvent ont confirmé les résultats précédents.

Pour l'instant, il semble (Melnitchenko et Trichina 1976, Fresnaye et LAVIE 1976) que ce sont les hybrides triples (reines hybrides de deux races fécondées par des mâles d'une troisième race) qui fournissent en moyenne les rendements les plus élevés. Ainsi, en France les meilleurs hybrides mis au point par Fresnaye ont une reine italo-caucasienne (mère ligustica, père caucasica) fécondée naturellement par des mâles de la race locale mellifica. Si l'intérêt de telles colonies ne peut-être mis en doute, il faut bien admettre que leur diffusion risque d'entrainer une pollution génétique de la population d'abeilles locales. Or plusieurs raisons font qu'il est souhaitable de limiter au maximum cette pollution:

- l'introduction de colonies hybrides dans un secteur où la population était jusque là intacte, si elle est favorable à leurs possesseurs, défavorisera à plus ou moins long terme les autres apiculteurs qui auront dans leurs cheptels des hybrides incontrôlés.

- les populations locales constituent des réserves naturelles de gènes coadaptés qu'il faut à tout prix préserver pour ne pas se trouver dans quelques années dans la situation où l'on se trouve actuellement pour certaines espèces ultra-sélectionnées.

- de toute façon, la fabrication même de ce type d'hybride repose sur la disponibilité de mâles de race locale.

La pollution génétique a lieu essentiellement par les mâles issus des reines hybrides, le contrôle des reines ne posant pas de difficultés et présentant une sécurité suffisante. Un obstacle mécanique à la sortie des mâles sans gêne pour les ouvrières n'est guère aisé à mettre en œuvre. C'est pourquoi, nous avons recherché un contrôle génétique.

\section{I. - PRINCIPE DE LA MÉThode}

Ce principe repose sur l'utilisation de mutations aptes à empêcher les mâles à féconder naturellement les reines. Les conditions suivantes doivent être requises :

$\left.1^{\circ}\right)$ Il faut qu'il y ait un maximum de mâles (issus d'une reine hybride) porteurs d'une mutation au moins. 
$2^{\circ}$ ) La reine hybride doit être parfaitement normale car elle doit être fécondée naturellement et doit pondre normalement.

$3^{\circ}$ ) Les ouvrières de cette reine hybride doivent être, elles aussi, parfaitement normales car elles assurent la récolte.

Considérons d'abord l'hypothèse d'une seule mutation notée $\mathrm{m}$. Trois cas seulement sont possibles pour une reine : elle est soit $(\mathrm{m} / \mathrm{m})$ soit $(\mathrm{m} /+)$, soit $(+/+)$.

Si elle est hétérozygote, il n'y aura que la moitié de sa descendance mâle qui portera la mutation. Pour remplir les conditions 2 et 3 , il suffit de supposer la mutation récessive ce qui compte tenu de ce que l'on sait sur les mutations déjà connues chez l'Abeille (RutTNER 1968) n'est pas trop contraignant. La pollution génétique sera divisée par deux ce qui représente un progrès certain mais qu'on peut juger insuffisant. Si la reine est homozygote $(\mathrm{m} / \mathrm{m})$, la condition 1 est parfaitement remplie et la pollution génétique est nulle. La condition 3 sera remplie si la mutation est récessive. Mais la condition 2 pose le problème de la recherche d'une mutation n'affectant que le sexe mâle. Bien qu'à priori, l'existence d'une telle mutation n'est pas impensable, sa découverte n'a pas encore été faite à notre connaissance. Nous abandonnerons donc pour l'instant l'hypothèse d'une seule mutation.

Envisageons l'hypothèse de deux mutations ( $\mathrm{m} 1$ et $\mathrm{m} 2$ ) entraînant séparément l'inaptitude des mâles à féconder naturellement les reines.

La reine hybride est donc porteuse des deux mutations. La condition 2 est résolue si la reine est hétérozygote pour les deux mutations et si celles-ci sont récessives. Si elles sont récessives, la condition 3 est, elle aussi, remplie puisque les mâles fécondant naturellement la reine hybride ne portent aucune de ces mutations (la possession de l'une les empêcherait de féconder). Calculons la proportion de mâles fécondants dans la descendance des reines hybrides:

+ si les mutations sont situées à des loci indépendants il y aura :

un quart de mâles $\mathrm{m} 1$

un quart de mâles $\mathrm{m} 2$

un quart de mâles $\mathrm{m} 1 \mathrm{~m} 2$

un quart de mâles normaux.

+ si les loci sont liés avec un pourcentage de recombinaison $r(0<r<1 / 2)$ deux cas sont à envisager selon la position respective des gènes mutés :

a) les gènes mutés sont sur le même chromosome. Le tableau I indique que la proportion de mâles normaux est de (1-r)/2 donc comprise entre $1 / 4$ et $1 / 2$.

b) les gènes mutés sont en opposition.

La proportion de mâles normaux (Cf. tableau I) est alors de $\mathrm{r} / 2$ donc comprise entre 0 et $1 / 4$.

Cette dernière éventualité apparaît donc comme la plus intéressante. Résumons là : la reine hybride doit être porteuse de deux mutations récessives et gouvernées par des gènes liés et en opposition. La fréquence des mâles normaux sera d'autant plus faible que la distance entre les deux gènes sera plus faible. 
TABL. 1. - Fréquences des divers génotypes de mâles fils de reines hétérozygotes à 2 loci liés (pourcentage de recombinaison égal à r).

TAB. 1. - Frequenz der verschiedenen Genotypen von Drohnen, die von Königinnen, heterozygot für 2 gekoppelte Loci, abstammen (Prozentsatz der Rekombination $=r$ ).

\begin{tabular}{|c|c|c|c|}
\hline & $\mathrm{m}_{1} \quad \mathrm{~m}_{2}$ & & $\mathrm{~m}_{1}+$ \\
\hline Königin & $+\quad+$ & Königin & $+m_{2}$ \\
\hline $\begin{array}{c}\text { mâles } \\
\text { Drohnen }\end{array}$ & $\begin{array}{c}\text { fréquence } \\
\text { Frequenz }\end{array}$ & $\begin{array}{c}\text { mâles } \\
\text { Drohnen }\end{array}$ & $\begin{array}{l}\text { fréquence } \\
\text { Frequenz }\end{array}$ \\
\hline $\mathrm{m} 1 \quad \mathrm{~m} 2$ & $1-r$ & $\mathrm{ml}+$ & $1-r$ \\
\hline & 2 & & 2 \\
\hline+ & $1-r$ & $+\mathrm{m} 2$ & $1-r$ \\
\hline & 2 & & 2 \\
\hline $\begin{array}{r}\mathrm{m} 2 \\
\end{array}$ & $\mathrm{r}$ & $\mathrm{m} 1 \quad \mathrm{~m} 2$ & $\mathrm{r}$ \\
\hline & 2 & & 2 \\
\hline $\mathrm{m} 1+$ & $\mathrm{r}$ & & $r$ \\
\hline & 2 & & 2 \\
\hline
\end{tabular}

II. - OBTENTION DE LA REINE HYBRIDE

Pour que les deux gènes mutés soient en opposition chez la reine hybride, il faut que les deux gamètes dont elle est issue soient porteurs chacun d'une mutation. Du côté paternel il n'y a pas de question : les mâles pères haploïdes ont le phénotype et le génotype mutant. Du côté maternel par contre on a le choix entre une femelle hétérozygote et une femelle homozygote, l'une et l'autre fournissant des gamètes porteurs de la mutation. Mais, la reine hybride étant de phénotype normal, il est impossible de connaitre son génotype si l'on n'est pas sûr du génotype des gamètes qui lui ont donné naissance. Il est donc nécessaire que sa mère (la génitrice) soit homozygote pour la mutation.

L'introduction des mutations dans les deux lignees parentales est un probleme classique qui est rendu très facile chez l'Abeille du fait de l'haploïdie des mâles. La figure 1 indique un moyen d'introduire une mutation à l'état homozygote chez une reine tout en conservant en moyenne $97 \%$ du génome de la souche d'origine. Si l'on ne considère que l'apparentement par la reine $F 1$, le cœfficient de consanguinité de cette reine mutante est de $1 / 512$ donc parfaitement négligeable. Un tel plan porte sur 6 générations pour atteindre la génitrice de la reine hybride. Sous nos conditions, il faut compter au moins deux ans pour y parvenir. 


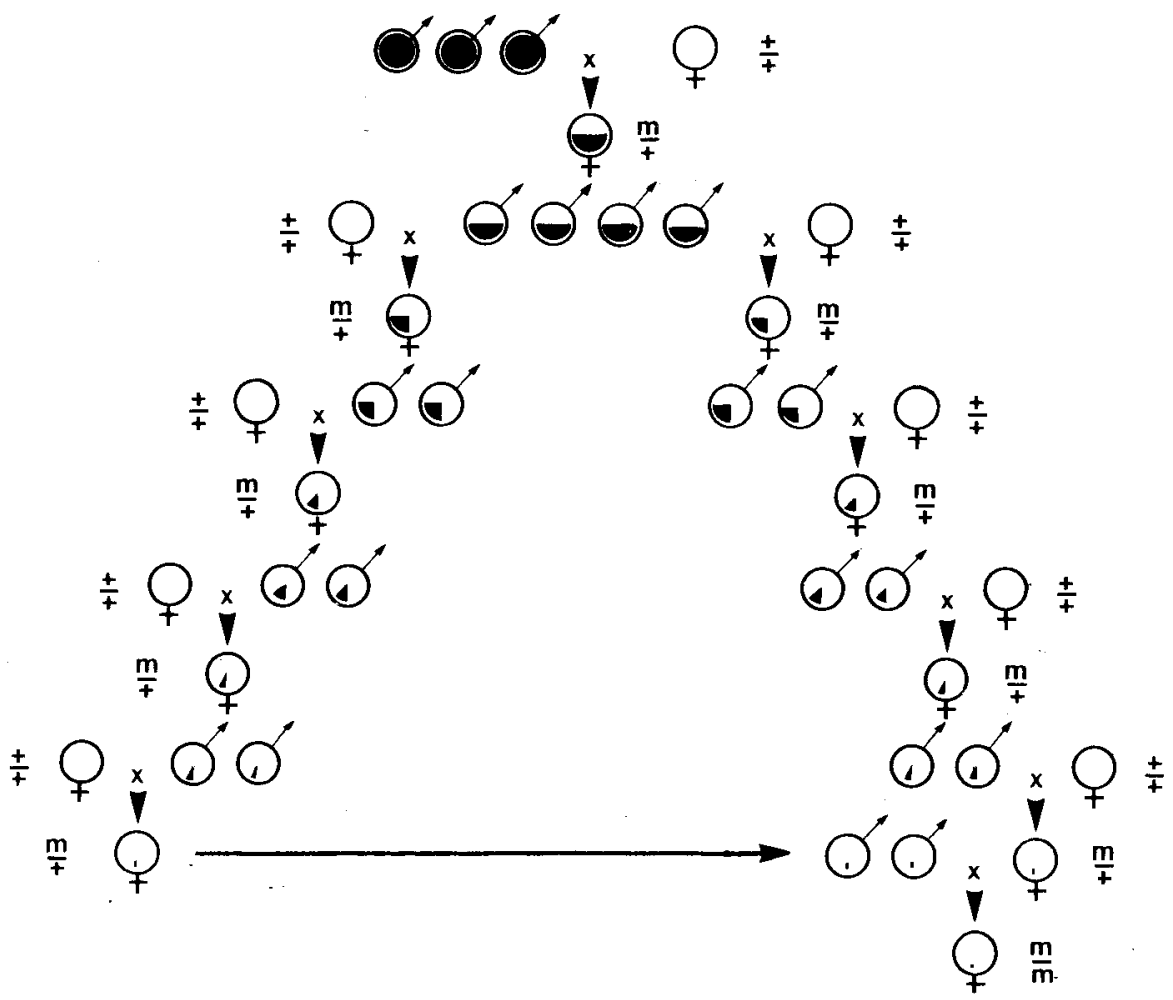

FIG. 1. - Schéma de croisements pour introduire un allèle mutant $(m)$ dans une souche.

En noir, est figuré la part de génôme de la souche porteuse de la mutation. Tous les mâles du schéma portent la mutation.

Le dernier croisement permet d'obtenir des reines homozygotes $(\mathrm{m} / \mathrm{m})$.

Авв. 1. - Kreuzungsschema für den Einbau eines mutierten Allels $(m)$ in einem Zuchtstamm.

Das Genom des Stammes, der die Mutation trägt, ist schwarz dargestellt.

Alle Drohnen des Schemas besitzen die Mutation. Bei der letzten Kreuzung erhält man homozygote Königinnen $(\mathrm{m} / \mathrm{m})$.

\section{III. - CHOIX DES MUTATIONS}

Quels critères doivent remplir les deux mutations?

1. - elles doivent être récessives.

2. - les gènes doivent être liés et le plus proche possible.

3. - elles doivent chacune empêcher les mâles de féconder naturellement les reines.

4. - elles ne doivent pas affecter les organes génitaux des deux sexes, altérer la qualité des gamètes mâles et femelles qui doivent rester fécondants et ne pas modifier le comportement de ponte de la reine.

A priori il semble qu'il existe deux mutations remplissant les conditions cidessus : "pearl " et "cream ". En effet, elles sont récessives et étroitement liées : le pourcentage de recombinaison est de $0,33 \%$ (LAIDLAW et al. 1965). Les homozygotes 
sont aveugles donc incapables de se repérer en vol et de s'accoupler naturellement. Par contre, l'insémination artificielle ne pose pas de problème et la reine homozygote ne devrait pas avoir de difficulté à pondre. Les mutations pearl et cream interviennent dans la chaîne de synthèse des ommochromes des yeux composés. DustmanN (1969) a montré que les mutants pearl et cream étaient incapables d'effectuer la granulation de la 3-hydroxykynurénine au niveau des cellules pigmentaires de l'œil. Deux autres mutations intervenant dans la même chaîne de synthèse snow' et chartreuse ont un effet pléïotrope plus ou moins accusé sur certaines caractéristiques neurophysiologiques (Ponomarenko 1976) et il faut noter que si ces mutations sont récessives au niveau de la coloration oculaire, il n'en va pas obligatoirement de même pour tous les autres caractères affectés par elles. Ainsi la mutation snow apparait dominante pour le temps nécessaire à l'installation de la narcose à l'éther et semi dominante pour le rythme de la danse. Par contre les mêmes tests réalisés avec la mutation chartreuse montrent des différences plus faibles entre les trois catégories $(\mathrm{ch} / \mathrm{ch}, \mathrm{ch} /+,+/+)$ et une récessivité plus générale. Il convient donc d'être prudent dans l'utilisation des mutations cream et pearl et de vérifier que leurs effets sur l'ensemble des caractéristiques des abeilles et spécialement à l'état hétérozygote ne constituent pas un obstacle determinant à leur emploi.

Dans le cas favorable où leur utilisation est possible, en suivant la méthode que nous proposons, la pollution génétique résultant de l'emploi de reines hybrides serait divisée par 600 .

Reçu pour publication en mai 1978. Eingeganger im Mai 1978.

\section{ZUSAMMENFASSUNG}

Mehrere Autoren haben auf die Bedeutung der Schaffung von Hybriden zwischen geographischen Bienenrassen zur Steigerung des Ertrages der Völker hingewiesen. Die besten Resultate wurden mit Dreifachhybriden erzielt : Die Königin, abstammend von Eltern verschiedener Rassen (also ein einfacher $\mathrm{Hy}$ bride), wurde mit Drohnen einer dritten Rasse gepaart. Aber unter bestimmten Umständen kann die Verwendung solcher Hybriden durch eine genetische Verunreinigung zur Schädigung der lokalen Bienenpopulation führen.

Um diese Verunreinigung auf ein Minimum zu beschränken, wird ein Verfahren untersucht, bei dem die von den Hybridköniginnen erzeugten Drohnen infolge des Besitzes einer Mutation zur natürlichen Paarung unfähig sind. Eine Prüfung der Liste der gegenwärtig bekannten Mutationen ergibt. dass dieses Ziel mit einer einzigen Mutation nicht zu erreichen ist. Erst zwei miteinander gekoppelte rezessive Gene können dazu führen, dass die genetische Pollution in erheblichem Ausmass verringert wird: diese beiden Gene müssen bei der Hybridkönigin heterozygot, und dazu in Oppositionsstellung (je eine Mutation auf einem der beiden Chromosomen) vorhanden sein. Um das zu erreichen, muss die Mutter der Hybridköni. gin für eine Mutation homozygot sein und instrumentell mit Drohnen der anderen Mutation gepaart werden.

Die Auswahl der Mutation muss folgenden Kriterien entsprechen : Rezessivität. enge Koppelung zwischen den beiden Genen, Verhinderung der natürlichen Paarung bei den Drohnen. aber im heterozygoten Zustand kein Einfluss auf die Fruchtbarkeit oder auf das Legeverhalten der Königin. Die beiden Mutationen "pearl" und "cream" (beide verursachen das Merkma! "weisse Augen") scheinen diese Bedingungen zu erfüllen. ihre enge Koppelung miteinander (Rekombinationswert $0.33 \%$ ) hat zur Folge. dass eine Hybridkönigin nur einen normalsichtigen Drohn unter 600 hervorbringt. Es muss allerdings 
noch geprüft werden, ob diese Mutation im heterozygoten Zustand nicht einen ungünstigen Einfluss auf den übrigen Stoffwechsel hat, so wie dies kürzlich für zwei Mutationen ("snow" und "chartreuse ") nachgewiesen worden ist, die ebenfalls in die Ommochromsynthese eingreifen.

\section{BIBLIOGRAPHIE}

Bornus L., 1972. - Résultats de l'examen comparatif de la valeur économique du croisement de certaines races d'abeilles. Symposium international Lunz am See. Août 1972, p. 87-89.

DustmanN. - Eine chemische Analyse der Augenfarbmutanten von Apis mellifica. J. Insect. Physiol., 1969,15, p. $2225-2238$.

Fresnaye J., et LAvie P., 1976. - Sélection et hybridation de l'abeille en France. Symposium de biologie de l'Abeille. Moscou Août 1976, p. 235-241.

Fresnaye J., Lavie P. et Boesiger E., 1974. - La variabilité de la production du miel chez l'Abeille de race noire (Apis mellifica mellifica) et chez quelques hybrides interraciaux. Apidologie, 5 (1) p. 1-20.

Laidlaw H. H., El Bandy M. A., et Tucker K. W., 1965. - Further linkage studies in the honeybee. $J$. Hered, 56, p. 39-41.

Melnitchenko A. N., et Trichina A. S., 1976. - Les bases écologiques et génétiques de l'hétérosis dans les colonies d'abeilles mellifères. Symposium de biologie de l'Abeille. Moscou. Août 1976, p. 225 232.

Ponomarenko V. V., 1976. - L'utilisation des mutants à manifestation chimique connue dans l'étude de la génétique de l'activité de signalisation et des caractères morphologiques. Symposium de biologie de l'Abeille. Moscou. Août 1976, p. 78-82.

RUTTNER F., 1968. - Methods of breeding honey-bees : Intra-racial selection or inter-racial Hybrids? Bee World, 49 (2) p. 66-72.

Velitckoov V. N., 1976. - Testage des hybrides inter-raciaux des abeilles. Symposium de biologie de l'Abeille. Moscou. Août 1976, p. 232-235. 